\title{
Impact of Internet of Things (IoT) in Smart Agriculture
}

\author{
O. Vishali Priya ${ }^{a, 1}$, Dr.R. Sudha ${ }^{a}$ \\ ${ }^{a}$ Department of Computer Science,PSG College of Arts \& Science, Coimbatore, \\ Tamilnadu, India
}

\begin{abstract}
In today's world, technology is constantly evolving; various instruments and techniques are available in the agricultural field. And within the agrarian division, the IoT preferences are Knowledge processing. With the help of introduced sensors, all information can be gathered. The reduction of risks, the mechanization of industry, the enhancement of production, the inspection of livestock, the monitoring of environment conditions, the roboticization of greenhouses, and crop monitoring Nearly every sector, like smart agriculture, has been modified by Internet-of-Things (IoT)-based technology, which has shifted the industry from factual to quantitative approaches. The ideas help to link real devices that are equipped with sensors, actuators, and computing power, allowing them to collaborate on a task while staying connected to the Internet, dubbed the "Internet of Things" (IoT). According to the World Telecommunication Union's Worldwide Guidelines Operation, the Internet of Things (IoT) is a set of sensors, computers, software, and other devices that are connected to the Internet. The paper is highly susceptible to the consequences of its smart agriculture breakthrough.
\end{abstract}

Keywords.: Internet of Things, Smart Farming, Smart Agriculture, Application of IOT, Benefits of Agriculture, Implications of implementation, Working of Agriculture

\section{Introduction}

This theory stems from advancements including the Internet of Things, Big Data, and Cloud Computing, among others, which have given rise to the concept of smartness. Farming IoT is a network of monitors, cameras, and computers that can all function together to help a farmer perform his job more effectively. This computer would be self-sufficient so that they will be able to interact with one another without the need for human intervention. To put it another way, the gadgets are pre-programmed with the knowledge of the moment and the motives for communicating with other instruments in the scheme.

Several agricultural industries have switched to IoT engineering for smart farming to increase productivity, performance, global market, and other features such as minimal human interference, time, and cost, among others. The advancement in technology ensures that sensors are becoming smaller, more modern, and more affordable. The

${ }^{1}$ O. VISHALI PRIYA, Department of Computer Science, PSG College of Arts \& Science, Coimbatore, Tamilnadu, India

Email: vishpriya2804@gmail.com. 
systems are also easily accessible and all-inclusive, allowing smart farming to be carried out with complete confidence. The answer to the challenges that this sector is currently experiencing is keen cultivation, which focuses on empowering advancement to agriculture. Much of this can be achieved with the aid of mobile phones and IoT gadgets. A farmer may obtain any need data or information, as well as monitor his agricultural field.

\section{Internet of Things (IoT)}

The Internet of Things (IoT) is the most productive and essential methodology for designing solutions to problems. IoT grows from a number of building blocks, such as sensors, applications, network elements, and other electronic devices. Furthermore, it improves the effectiveness of knowledge. IoT allows data to be exchanged across a network without the need for human intervention.

In the Internet of Things, be prepared to converse with objects in a natural fashion, rather than in the manner of a regular human being, such as a sensor, a car driver, and so on. This object has been given an address so that it can transmit data across a network. According to Gather's study, there would be a $30 \%$ increase in connected computer checks by the end of 2016 relative to 2015 . He predicts that by 2020 , this figure will rise to 26 billion.[1].

Because of the following considerations, IoT technology is more efficient:

- Access to the internet from every laptop.

- As little physical effort as possible

- Improved Access

- Time Management

- Communication that effective

\section{Smart Agriculture Using IoT}

Agriculture is India's economic development's most important pillar. Climate change is the most important boundary that conventional farming faces. Overwhelming flooding, the most extreme hurricanes and warm winds reduced rainfall, and other climatic shifts are among the many consequences. As a consequence of these considerations, performance suffers greatly. Climate change often has natural effects, such as periodic shifts in plant lifecycles.

In order to increase productivity and reduce boundaries in the farming sector, creative creativity and Internet of Things strategies were needed. The Internet of Things (IoT) is now shifting its focus to the agricultural sector, enabling farmers to face the immense obstacles they face. Using IoT, farmers may gain access to a wealth of data and knowledge regarding future trends and innovation. 
Since global agriculture is becoming more industrialized, it is critical to establish agricultural interdepartmental cooperation at the same time. Agricultural intercolumniation has resulted in a positive change in global agriculture.

As far as agricultural improvement is concerned, rural intercolumniation can be a major impediment to agricultural progress and reform and a cornerstone for sustaining stable and sustainable economic growth. We've been concentrating on agricultural data gain and framework improvement for a while now. Exceptional results had been shown in rural system improvement after several years of challenging efforts [2].

Smart agriculture is a broad term that refers to agricultural and food production practises that make use of IoT, big data, and advanced analytics. The Internet of Things refers to the integration of sensing, automation, and analytics technology into present agricultural processes. The most common IoT applications in smart agriculture are as follows:

$\checkmark$ Sensor-based systems for monitoring crops, soil, fields, animals, storage facilities, and just about anything else that has an impact on productivity.

$\checkmark$ Smart agricultural vehicles include drones, autonomous robots, and actuators.

$\checkmark$ Connected agricultural settings include smart greenhouses and hydroponics.

$\checkmark$ Data analytics, visualization, and management systems.

The Internet of Things, like other industries, offers hitherto impossible efficiency, resource and expenditure savings, automation, and data-driven operations in agriculture. However, in agriculture, these benefits aren't improvements; they're cures for an entire sector beset by a host of serious problems [3].

\subsection{Extremely efficient}

Agriculture is become a competition. With deteriorating soil, dwindling land supplies, and increasing weather unpredictability, farmers must produce more. IoT-enabled agriculture allows farmers to monitor their products and conditions in real time. They have fast perceptions, can predict issues before they arise, and make well-informed decisions on how to avoid them. Agriculture IoT solutions include automated features such as demand-based watering, fertilisation, and robot harvesting.

\subsection{Expansion}

By the time we reach 9 billion people, $70 \%$ of the world's population will be living in cities. IoT-based greenhouses and hydroponic systems, which should be able to feed these folks with fresh fruits and vegetables, enable short food supply chains. Thanks to sophisticated closed-cycle agricultural systems, food may be produced in supermarkets, on the walls and rooftops of buildings, in shipping containers, and, of course, in the comfort of everyone's home.

\subsection{Resources are in short supply.}

Many agricultural IoT solutions seek to make the most efficient use of resources like water, power, and land. Precision farming is based on data acquired from a variety of sensors in the field, allowing farmers to precisely allocate exactly adequate nutrients to one plant. 


\subsection{Hygienic Procedure}

Smart farming using IoT is a tried-and-true method of reducing pesticide and fertiliser consumption. Precision farming not only saves water and energy and makes farming more ecologically friendly, but it also drastically decreases the need of pesticides and fertilisers. In compared to traditional agricultural methods, this technique yields a cleaner, more organic end product.

\subsection{Agility}

One of the benefits of using IoT in agriculture is the increased adaptability of operations. Thanks to real-time monitoring and forecasting technologies, farmers can react quickly to any significant change in weather, humidity, air quality, or the condition of any crop or soil in the field. Agriculture specialists can now save crops in the face of extreme weather changes thanks to new skills.

\subsection{The quality of the product has improved.}

Data-driven agriculture aids in the production of more and better commodities. Using soil and crop sensors, overhead drone monitoring, and farm mapping, farmers may gain a better understanding of the complex relationships between the environment and the quality of their crops. Using connected systems, they can replicate ideal conditions and increase the nutritional value of the goods.

The whole handle revolves around the gathering of data for farmers and other collaborators to use. Usually, the most critical feature of the activity. Sensors, microphones, and fawning pictures are among the gadgets included. The second segment consists of an agreement that will assist in sharing knowledge provided by the machines, as previously mentioned. Various types of network advancements, such as GSM, LTE, Wi-Fi, and 3G, may be used based on connectivity and requirements. The third section, similar to Cloud administrations, consists of data processing and computing creativity.

Cloud servers may be made open without any limitations, rendering them suitable for IoT frameworks. On such servers, knowledge may be stored and processed. Cloud administrations may be accessed on a pay-per-use basis, although they are becoming more common for this purpose. The system's final component will be the Big Data analytics tools, which will sift through the vast amounts of data generated and stored on cloud storage to find important trends and patterns.

The steps in working of Agriculture IOT:

Smart mobile data collection

Network-based data transfer

Cloud-based data collection and computing

Use large data software to analyze data 


\section{Major Applications}

Each aspect of conventional farming strategy can be modified on a very simple level by implementing the most recent detecting and IoT advances in agriculture practices. Right now, consistent convergence of wireless sensors and the Internet of Things in smart agriculture will propel agriculture to previously unimaginable heights[4]. IoT may help advance the solutions to various typical farming problems, such as dry spell reaction, surrender optimization, arrive reasonableness, water method, and bother regulation, by adopting the tenets of savvy agriculture [5].

4.1. Climate Change: Agriculture is significantly impacted by climate change. Furthermore, a lack of understanding of climate has a substantial influence on agricultural production quantity and quality. IoT technologies, on the other hand, allow you to track weather conditions in real time. Sensors have been deployed both within and outside of agriculture regions. They collect data from the environment, which is then used to determine which crops are most suited to growing and surviving in the given climatic conditions. Sensors are used across the Internet of Things ecosystem to accurately monitor real-time weather factors such as humidity, rainfall, temperature, and more. There are a variety of sensors available to monitor and customise all of these factors to fit your smart farming demands. These sensors monitor the health of the crops as well as the weather conditions around them. When unexpected weather conditions are found, an alarm is dispatched. The necessity for human presence during inclement weather is removed, boosting output and allowing farmers to enjoy additional agricultural advantages.

4.2. Precision Farming: One of the most well-known IoT applications in agriculture is precision farming, often known as precision agriculture. Animal monitoring, vehicle tracking, field observation, and inventory monitoring are examples of smart farming applications that serve to make farming more precise and controlled. The goal of precision farming is to assess data supplied by sensors and respond appropriately. Precision farming allows farmers to collect data from sensors and analyse it to make educated and timely decisions. Irrigation management, livestock management, vehicle tracking, and other precision agricultural procedures are all vital in increasing efficiency and effectiveness. Precision farming allows you to evaluate soil conditions as well as other pertinent data in order to increase operational efficiency. Not only that, but you may also check water and nutrient levels by monitoring the associated devices' real-time operational conditions.

4.3. Smart Greenhouse: The Internet of Things has allowed weather stations to change climatic conditions automatically in response to a set of instructions, allowing us to make our greenhouses smart. In greenhouses, the integration of IoT has eliminated the need for human intervention, making the entire process more costeffective while also increasing accuracy. Solar-powered Internet of Things sensors, for example, may be utilised to build modern, low-cost greenhouses. These sensors collect and transmit real-time data, allowing for precise real-time greenhouse monitoring. Thanks to the sensors, the water use and greenhouse state may be tracked via emails or SMS messages. Irrigation is carried out automatically and intelligently via the Internet of Things. Pressure, humidity, temperature, and light levels may all be measured with these sensors. 
4.4. Data Analytics: IoT device data necessitates more storage than a conventional database system can supply. Cloud-based data storage and an end-to-end IoT platform are critical components of the smart agriculture system. These systems are expected to play a key role in facilitating the execution of better tasks. In the IoT era, sensors are the primary source of large-scale data collecting. The data is analysed and transformed into valuable information using analytics tools. Data analytics may be used to analyse weather, livestock, and agricultural conditions. Using technological improvements, the knowledge obtained enables for better decision-making. You may learn about the real-time status of your crops by collecting data from sensors utilising IoT devices. Predictive analytics can help you gain insight into harvesting decisions and make better ones. Farmers can utilise trend analysis to forecast upcoming weather and crop harvesting circumstances. IoT has assisted farmers in preserving crop quality and soil fertility, resulting in increased production volume and quality in the agriculture business.

4.5. Aerial Drones in Agriculture: Agricultural operations have almost totally altered as a result of technology advancements, with the most recent disruption being the introduction of agricultural drones. Drones are used for agricultural health checks, crop monitoring, planting, crop spraying, and field analysis on the ground and in the air. Thanks to suitable strategy and planning based on real-time data, drone technology has given the agriculture industry a boost and makeover. Drones with thermal or multispectral sensors detect areas where irrigation has to be adjusted. As the crops develop, sensors assess their health and calculate their vegetation index. The environmental impact of smart drones was finally reduced. As a consequence, the quantity of chemical that reaches the groundwater has decreased significantly [6].

Agriculture facilitated by the Internet of Things has assisted in the adoption of cuttingedge technology solutions to age-old knowledge. This has helped to close the gap in output, quality, and yield. Quick reaction and minimum crop damage are ensured by data obtained by collecting and importing data from multiple sensors for real-time use or storage in a database. Because to end-to-end intelligent operations and improved business process execution, produce is processed faster and reaches supermarkets in the shortest time feasible [7].

\section{Implications of Implementation}

Agriculture IoT implementation on a wide scale is possible, as it were, with the government's support [8]. It will aid in the decision-making process by delivering userfriendly plans and strategies. It can have subsidized gadgets and bases that farmer can't handle in any other way. Agriculture supply chain flaws must be addressed [9]. To offer the most severe benefits to farmers and consumers, the position of an agent should be re- examined and handled [10].

\subsection{The apparatus}

To build an IoT solution for agricultural, you must first choose the sensors for your equipment (or create a custom one). The kind of data you want to collect and the ultimate objective of your solution will guide your selection. In any case, the precision and dependability of the data obtained will decide the success of your product, therefore the quality of your sensors is important. 


\subsection{The brain}

Data analytics should be part of any smart agriculture solution. It'll be pointless if you can't make sense of the data you've acquired. You'll require advanced data analytics abilities, as well as prediction algorithms and machine learning, to derive valuable insights from the acquired data.

\subsection{Performing routine maintenance}

Maintaining your hardware is a big challenge for IoT devices in agriculture because sensors are frequently used in the field and can be easily destroyed. As a result, you must make certain that your hardware is both durable and repairable. You'll have to replace your sensors more frequently than you'd want if you don't.

\subsection{Movement ability}

Agricultural applications should be particularly built for use in the field. For a business owner or farm manager, the information should be available on-site or remotely by smartphone or desktop computer.Each linked device should also be self-contained and have enough wireless range to communicate with other devices as well as provide data to the central server.

\section{5 smart-farming-app-development infrastructure.}

To ensure that your smart farming application functions well, you'll need a solid internal architecture (and can manage the data load). Furthermore, your internal systems must be protected. If you fail to effectively safeguard your system, breaking in, stealing your data, or even gaining control of your autonomous tractors becomes more possible.

\subsection{Interdependence}

The necessity for data to be sent across several agricultural locations continues to be a roadblock to smart farming adoption. Naturally, the connectivity connecting these facilities must be dependable enough to withstand adverse weather and offer continuous service [11]. Despite current efforts to develop universal standards in this area, IoT devices currently use a range of communication protocols. The development of 5G and technologies like space-based Internet will, hopefully, help to resolve this issue.

\subsection{The amount of data collected on a regular basis}

Because there are so many different forms of data in the agriculture industry, finding the optimal data collection frequency may be challenging. Data from field-based, aerial, and environmental sensors, applications, devices, and equipment, as well as processed analytical data, are all subject to restrictions and laws. The secure and timely delivery of this data, as well as its interchange, is one of the most difficult aspects of smart farming.

\section{Conclusion}

As a result, smart agriculture is needed. The Internet of Things would assist in the development of smart agriculture. IoT is used in various agricultural domains to 
improve time efficiency, water conservation, crop monitoring, soil management, bug spray and pesticide safety, and so on. It also eliminates human labor, deconstructs agricultural methods, and creates a difference in smart farming implementation. Agriculture is a profession that has relied on traditional practices and experiences up to now. However, the passage of time has affected rural traditions, and they have started to adapt to the flow of change. The usage of the Internet of Things (IoT) in agriculture would increase yields and help control all cultivate operations. Agriculture must undergo significant improvements since a substantial portion of the population relies on it for survival.

\section{Reference}

[1] Babu Loganathan, Ganesh. "Smart Agriculture System With E-Carbage Using Iot." International Journal of Modern Agriculture 10, no. 1 (2021): 928-931.

[2] Haseeb, Khalid, Ikram Ud Din, Ahmad Almogren, and Naveed Islam. "An energy efficient and secure IoT-based WSN framework: An application to smart agriculture." Sensors 20, no. 7 (2020): 2081

[3] Miles, Badreddine, El-Bay Bourennane, Samia Boucherkha, and Salim Chikhi. "A study of LoRaWAN protocol performance for IoT applications in smart agriculture." Computer Communications 164 (2020): 148-157.

[4] Ratnaparkhi, Sanika, Suvaid Khan, Chandrakala Arya, Shailesh Khapre, Prabhishek Singh, Manoj Diwakar, and Achyut Shankar. "Smart agriculture sensors in IOT: A review." Materials Today: Proceedings (2020).

[5] Kassim, Mohamed Rawidean Mohd. "IoT Applications in Smart Agriculture: Issues and Challenges." In 2020 IEEE Conference on Open Systems (ICOS), pp. 19-24. IEEE, 2020.

[6] Kumar, Dr A. Senthil, Dr AR, L. Ganesh Babu, and Dr G. Suresh. "Smart Agriculture Robo With Leaf Diseases Detection Using IOT." European Journal of Molecular \& Clinical Medicine 7, no. 11 (2022): 2462-2469.

[7] Marcu, Ioana, George Suciu, Cristina Bălăceanu, Alexandru Vulpe, and Ana-Maria Drăgulinescu. "Arrowhead technology for digitalization and automation solution: smart cities and smart agriculture." Sensors 20, no. 5 (2020): 1464.

[8] Rasooli, Mohammad Wasi, Brij Bhushan, and Nagesh Kumar. "Applicability of wireless sensor networks \& IoT in saffron \& wheat crops: A smart agriculture perspective." Int. J. Sci. Technol. Res 9, no. 2 (2020): 2456-2461.

[9] Prasad, Shitala, Sateesh K. Peddoju, and Debashis Ghosh. "Energy efficient mobile vision system for plant leaf disease identification." In 2014 IEEE Wireless Communications and Networking Conference (WCNC), pp. 3314-3319. IEEE, 2014.

[10] Dhaware, Chaitali G., and K. H. Wanjale. "A modern approach for plant leaf disease classification which depends on leaf image processing." In 2017 International Conference on Computer Communication and Informatics (ICCCI), pp. 1-4. IEEE, 2017.

[11] Singh, Vijai, and A. K. Misra. "Detection of unhealthy region of plant leaves using image processing and genetic algorithm." In 2015 International Conference on Advances in Computer Engineering and Applications, pp. 1028-1032. IEEE, 2015. 